\title{
Glycerol Monolaurate Enhances Reproductive Performance, Egg Quality and Albumen Amino Acids Composition in Aged Hens with Gut Microbiota Alternation
}

\author{
Tao Liu ${ }^{1}$, Chuang $\mathrm{Li}^{2}$, Yang $\mathrm{Li}^{1}$ and Fengqin Feng ${ }^{1,3, * \mathbb{C}}$ \\ 1 College of Biosystems Engineering and Food Science, Zhejiang University, Hangzhou 310058, China; \\ otaoliu@zju.edu.cn (T.L.); Ly3143@zju.edu.cn (Y.L.) \\ 2 School of Biological Science and Engineering, Hebei University of Science and Technology, \\ Shijiazhuang 050018, China; jiashuo0369@163.com \\ 3 Ningbo Institute of Zhejiang University, Ningbo 315100, China \\ * Correspondence: feng_fengqin@hotmail.com; Tel./Fax: +86-571-88982192
}

Received: 5 June 2020; Accepted: 17 June 2020; Published: 1 July 2020

check for updates

\begin{abstract}
As it is known to all that the laying rate of commercial laying hens is significantly decreased during the late laying period, paralleled with increased feed conversion rate (FCR) and declined egg quality. However, recent studies demonstrate that reproductive performance as well as egg quality in aged hens can be largely improved by feed supplements. Among them, glycerol monolaurate (GML), a monoglyceride of medium chain fatty acids, is regarded as a promising supplement in poultry production. The aim of this experiment was to evaluate the effect of dietary GML on laying performance, serum biochemical indices, egg quality and gut microbiota in hens during 40-64 weeks of age. A total of 378 (40-week-old) Hy-Line Brown laying hens were randomly assigned into three treatments ( 21 hens/replicate and 6 replicates/treatment). The control group received a basal diet, and the treated groups fed basal diets containing 150 and $300 \mathrm{mg} / \mathrm{kg} \mathrm{GML}$. The results revealed that laying rate $(p<0.05)$ was significantly increased and the FCR $(p<0.05)$ was reduced in GML-containing groups compared with the control group. The egg quality (including eggshell thickness and strength) was notably improved by GML supplementation at the later late laying period, and the content of total amino acids and flavor amino acids in the albumen were also increased. Inclusion of $300 \mathrm{mg} / \mathrm{kg}$ GML in diet increased the levels of laying-related sex hormones (serum follicle stimulating hormone, luteinizing hormone and estradiol, $p<0.05$ ), serum calcium and lipid (total serum cholesterol, triglycerides and low-density lipoprotein cholesterol, $p<0.05$ ). Moreover, the community and microbial function of gut microbiota were notably altered by GML supplementation. These findings revealed that dietary GML improved the reproductive performance and egg quality mainly by stimulating the sex hormones secretion, serum calcium metabolism and nutrients utilization, which provided us a new approach to simultaneously promote the egg production and improve the egg quality at aged hens.
\end{abstract}

Keywords: glycerol monolaurate; aged hens; reproductive performance; egg quality; amino acids

\section{Introduction}

After the end of the peak egg-laying period (around 40-48 weeks of age), the reproductive performance and egg quality are gradually decreased with increasing age of the hens [1,2]. During the late laying period, the egg production is declined, accompanied with decreased yolk synthesis and accumulation due to lowered sex hormone concentrations including follicle stimulating hormone 
(FSH), luteinizing hormone (LH) and estradiol (E2) in aged hens [3]. Moreover, there is increase in feed conversion ratio, caused by poor egg production and feed utilization. In parallel, the egg weight and size are increased, eggshell thickness and breaking strength, and Haugh unit (HU) are reduced as the hens aged, resulting in higher cracked egg rate (\% cracks), shortened egg storage time and poor flavor $[4,5]$. Therefore, continuously increasing concern is now focused on improving the reproductive performance, feed efficiency and egg quality of the late laying period hens. Presently, application of some supplements such as the rubber seed oil [6], peppermint [7], tea polyphenol [8], Lonicera confusa and Astragali Radix extracts [4] enhance the egg production, feed conversion rate (FCR), sensory attributes and nutritional quality in aged hens, which remind us that nutritional intervention may be an effective and natural approach to solve the problem.

Medium chain fatty acids (MCFAs) are a group of fatty acids with 6-12 C atoms extracted from edible fats such as coconut oil and milk fat by lipid fraction separation, which have shown positive effects on health, production and nutrient digestibility in poultry [9]. As a monoglyceride of MCFAs, glycerol monolaurate (GML), a naturally occurring glycerol monoester of lauric acid (C12:0), is recognized as a safe food emulsifier approved by the US Food and Drug Administration [10]. It has strong antibacterial effects especially against Gram positive bacteria by suppressing the growth and virulence of numerous bacteria, fungi and enveloped viruses in vitro [11]. Besides, GML can be utilized directly by the enterocytes for energy production and thereby helps to support the integrity of the intestinal tissue in broilers [12]. Recent research show that dietary GML improve the production performance, health, feed efficiency and quality in poultry, and is regarded as a promising feed supplements in production [13,14]. According to the results from Fortuoso et al. [13], GML supplementation increase the average body weight, feed consumption and carcass yield in male Cobb 500 broilers without toxic side effect. Likewise, broilers fed diet containing GML show improvements on body weight gain, feed efficiency, immunological and nutritional status in Ross 308 broilers [14]. Our laboratory demonstrated that dietary GML could improve reproductive performance, egg quality and feed efficiency in Hy-Line Brown laying hens during 44-52 weeks of age $[15,16]$.

Although the effect of dietary GML on reproductive performance, egg quality, serum biochemical indices and intestinal morphology in laying hens at 44-52 weeks of age were evaluated by our previous study [15]. However, this experimental period was too short to assess the overall impact of GML on the whole late laying period for hens. Moreover, the exact time of GML affecting the reproductive performance and egg quality warrants further study. Furthermore, GML has the strongest antimicrobial capacity among the medium chain 1-monoglycerides in vitro, but the effect of dietary GML on indigenous microbiota in laying hens remain unknown [11]. Therefore, this study was designed to evaluate the effect of GML supplementation on laying hens from the end of the peak laying period to the whole late laying period, including reproductive performance, feed efficiency, egg quality and gut microbiota. GML is a typical representative of medium chain fatty acids 1-monoglycerides (MGs), the findings in this study will comprehensively provide theoretical evidence for the efficacy of dietary MGs on egg production and quality control.

\section{Materials and Methods}

\subsection{Experiment Design}

A total of 378 (40-week-old) Hy-Line brown laying hens were randomly assigned into three treatments ( 21 hens/replicate and 6 replicates/treatment). Dietary treatments included the basal diet containing $0 \mathrm{mg} / \mathrm{kg}$ GML as the control group (CON, Table 1) and the basal diet containing $150 \mathrm{mg} / \mathrm{kg}$ (GML150) and $300 \mathrm{mg} / \mathrm{kg}$ GML (GML300). GML (95\% purity, CAS No. 142-18-7) was provided by Hangzhou Kangyuan Food Technology Co., Ltd. (Hangzhou, China) and was added into the diets by replacing the same energy amount of oil. The basal diet was formulated to meet the requirement of National Research Council 2017 for laying hens [17]. The feeding trial lasted for 24 weeks. Three hens housed in an individual cage $\left(40 \mathrm{~cm} \times 35 \mathrm{~cm} \times 60 \mathrm{~cm}\right.$, with a floor slope of $\left.12^{\circ}\right)$ equipped with 2 nipple 
drinkers and 1 feeder, and seven sequential cages fed with the same diet trough were arranged as a replicate, and all replicates were equally distributed in different spatial directions Hens were fed ad libitum twice daily at 5:00 a.m. and 1:00 p.m. and water nipples were available all times. All hens were housed in three-tier battery cages sharing a room with $25 \pm 2{ }^{\circ} \mathrm{C}, 60 \%-65 \%$ relative humidity, and a 16-h photoperiod. The protocol was approved by the Animal Care and Use Committee of Zhejiang University (No. ZJU-BEFS-2016004), Hangzhou, China.

Table 1. Composition and nutrient level of the basal diet for 2 sub-trials (as fed-basis).

\begin{tabular}{|c|c|c|}
\hline Ingredients, \% & 40-58 Week of Age & 58-64 Week of Age \\
\hline Corn & 62.90 & 64.90 \\
\hline Soybean meal & 23.55 & 21.48 \\
\hline Limestone & 7.93 & 7.93 \\
\hline Salt & 0.30 & 0.30 \\
\hline Fish oil & 0.03 & 0.03 \\
\hline Rapeseed oil & 0.59 & 0.66 \\
\hline${ }^{1}$ Premix & 4.70 & 4.70 \\
\hline Total & 100 & 100 \\
\hline \multicolumn{3}{|l|}{ Calculated/analyzed values } \\
\hline${ }^{2}$ Metabolizable energy (MJ/kg) & 11.85 & 11.98 \\
\hline${ }^{3}$ Crude protein, $\%$ & 16.10 & 15.80 \\
\hline Crude fat, $\%$ & 2.86 & 2.91 \\
\hline${ }^{3}$ Calcium, \% & 3.73 & 3.78 \\
\hline Total phosphorus, $\%$ & 0.60 & 0.57 \\
\hline Lysine, \% & 0.81 & 0.75 \\
\hline Methionine + Cysteine, $\%$ & 0.65 & 0.60 \\
\hline Methionine, $\%$ & 0.35 & 0.32 \\
\hline
\end{tabular}

${ }_{1}$ Provided the following per kilogram of diet: vitamin $\mathrm{A}, 10,000 \mathrm{IU}$; vitamin $\mathrm{D}_{3}, 4000 \mathrm{IU}$; vitamin $\mathrm{E}, 200 \mathrm{IU}$; vitamin $\mathrm{B}_{1}, 4 \mathrm{mg}$; vitamin $\mathrm{B}_{2}, 6 \mathrm{mg}$; vitamin $\mathrm{B}_{6}, 5 \mathrm{mg}$; vitamin $\mathrm{B}_{12}, 1 \mathrm{mg}$; vitamin $\mathrm{K}_{3}, 3 \mathrm{mg}$; biotin, $0.5 \mathrm{mg}$; folic acid, $3.0 \mathrm{mg}$; D-pantothenic acid, $20 \mathrm{mg}$; nicotinic acid, $20 \mathrm{mg}$; Cu, $10 \mathrm{mg}$; Fe, $100 \mathrm{mg}$; Mn, $100 \mathrm{mg}$; Zn, $100 \mathrm{mg}$; Se, $0.40 \mathrm{mg}$. ${ }^{2}$ Values were calculated according to metabolizable energy of feedstuffs for poultry provided by NRC $2017 .{ }^{3}$ The numbers were analyzed values.

\subsection{Reproductive Performance and Egg Quality}

The mortality, number and weight of the total eggs, broken eggs, soft eggshell eggs and misshapen eggs were recorded on a daily basis. Feed consumption was recorded weekly. For each replicate, average egg weight and broken rate were calculated on a weekly basis, and laying rate and feed conversion ratio (FCR) were calculated taking mortality into account.

At 48, 56 and 64 weeks of age, the eggshell thickness, eggshell strength, albumen height and Haugh units (HU) were determined using fresh eggs ( 5 eggs/replicate and 30 eggs/treatment) by a digital egg tester (DET 6000, NABEL Co., Ltd., Kyoto, Japan). The eggshell thickness was measured at the equatorial region using an eggshell thickness gauge (Mitutoyo, NABEL Co., Ltd., Kyoto, Japan).

\subsection{Sampling and Preparation}

At the end of 64 weeks of age, three randomly selected hens from each replicate ( 3 hens/replicate and 18 hens/treatment) were weighed and slaughtered after feed deprivation for $12 \mathrm{~h}$. Serum samples were collected from the jugular vein by vacuum tubes $(5 \mathrm{~mL})$ containing coagulant gel, and were obtained by centrifugation $\left(2000 \times \mathrm{g}\right.$ for $15 \mathrm{~min}$ at $\left.4{ }^{\circ} \mathrm{C}\right)$ and stored at $-80^{\circ} \mathrm{C}$ for further analysis. Cecal digesta was promptly sampled and frozen in liquid nitrogen, and transported to the laboratory in a dry-ice pack, then kept at $-80{ }^{\circ} \mathrm{C}$. Five eggs from each replicate ( 5 eggs/replicate and $30 \mathrm{eggs} /$ treatment) were randomly selected, and the albumen and yolk were separated and collected by replicate. The albumen and yolk were immediately frozen on dry-ice and freeze-dried (LGJ-10, Beijing Huaxing Technology Development Co., Ltd., Beijing, China), and then ground to pass through a 40-mesh sieve and stored at $-80^{\circ} \mathrm{C}$. 


\subsection{Serum Biochemical Indices and Sex Hormones}

Follicle stimulating hormone (FSH), luteinizing hormone (LH) and estradiol (E2) were measured using the commercial ELISA kit (Catalog number: JYM0055Ch (FSH), JYM0032Ch (LH) and JYM0049Ch (E2), Wuhan Colorful Gene biological technology Co., Ltd., Wuhan, China). Total serum cholesterol (TC), triglycerides (TG), high-density lipoprotein cholesterol (HDL-C), low-density lipoprotein cholesterol (LDL-C), alkaline phosphatase (AKP), calcium, glucose, total protein and total antioxidant capacity (T-AOC) were determined using kits from Nanjing Jiancheng Bioengineering Institute (Nanjing, Jiangsu, China) by following the manufacturer's instructions.

\subsection{Yolk Fatty Acids Profile}

Dry samples $(0.10 \mathrm{~g})$ were weighed into ultra-sound cleaned $15 \mathrm{~mL}$ tubes, then $3.2 \mathrm{~mL}$ formyl chloride (acetyl chloride: anhydrous methanol, 1:10; $v / v$ ) and $3.2 \mathrm{~mL}$-hexane containing $1 \mathrm{mg} / \mathrm{mL}$ $\mathrm{C} 17: 0$ (internal standard) were added prior to heating for $2 \mathrm{~h}$ at $75{ }^{\circ} \mathrm{C}$. After the tubes were cooled to room temperature, $4 \mathrm{~mL} 7 \%$ potassium carbonate $(v / v)$ was added and centrifuged for $5 \mathrm{~min}$ at $3000 \times g$, then the top layer was filtered through a $0.22-\mu \mathrm{m}$ membrane filter prior to gas chromatograph [18]. Analysis was conducted by a gas chromatography (GC-2014, Shimadzu Corporation, Tokyo, Japan) equipped with a column (DB-23, $60 \mathrm{~m} \times 0.25 \mathrm{~mm} \times 0.25 \mu \mathrm{m}$, Agilent, Santa Clara, CA, USA). Nitrogen at a constant flow of $0.65 \mathrm{~mL} / \mathrm{min}$ was the carrier gas. FID detector temperature and injector oven temperature was set at $250^{\circ} \mathrm{C}$ and $230^{\circ} \mathrm{C}$. The temperature program of the oven was: the initial oven temperature was $100^{\circ} \mathrm{C}$ and hold for $2 \mathrm{~min}$, then raised to $160^{\circ} \mathrm{C}$ at a rate of $10^{\circ} \mathrm{C} / \mathrm{min}$ and hold for $4 \mathrm{~min}$, then raised to $210^{\circ} \mathrm{C}$ at a rate of $10^{\circ} \mathrm{C} / \mathrm{min}$ and hold for $5 \mathrm{~min}$, then raised to $240^{\circ} \mathrm{C}$ at a rate of $10^{\circ} \mathrm{C} / \mathrm{min}$ and hold for $10 \mathrm{~min}$. Identification and quantification of fatty acids were aided with the use of certified reference standards (37 component Mix, Supelco 18919-1AMP, Sigma, St. Louis, MO, USA) and internal standard (C17:0).

\subsection{Albumen Amino Acids Profile}

Dry samples $(20 \mathrm{mg}$ ) were dissolved in $1 \mathrm{~mL} 6 \mathrm{M} \mathrm{HCl}$ in ultra-sound cleaned tubes under pure nitrogen atmosphere, then were heated for $2 \mathrm{~h}$ at $150{ }^{\circ} \mathrm{C}$ in a digestion furnace (SH60A, Jinan Hanon Instruments Co., Ltd., Jinan, China). Then, $10 \mu \mathrm{L}$ digested samples or standard amino acids solution (Supelco AA-S-18, Sigma, St. Louis, MO, USA) was pipetted into separated 1.5-mL microcentrifuge tubes and dried under vacuum. Thereafter, $20 \mu \mathrm{L}$ derivatization reagent (ethanol: water: triethylamine: phenyl-isothiocyanate, $7: 1: 1: 1, v / v / v / v)$ was added and kept sealed for 30 min at room temperature under nitrogen atmosphere. The reagents were then removed under vacuum [19]. The dried derivatized samples and standard amino acids were first dissolved in $50 \mu \mathrm{L}$ solvent $\mathrm{B}$ (water: methanol, 1:4, v/v) and mixed thoroughly. Then $450 \mu \mathrm{L}$ solvent $\mathrm{A}$ ( $20 \mathrm{mM}$ sodium acetate containing $0.1 \%$ triethylamine, $\mathrm{pH}=6.5$ ) was added and mixed thoroughly. The solution was filtered through a $0.22-\mu \mathrm{m}$ membrane filter prior to high performance liquid chromatography (Waters e2695, Waters Corporation, Milford, MA, USA). Ten-microliter was injected into the column (Ultimate AQ-C18, $4.6 \mathrm{~mm} \times 250 \mathrm{~mm} \times 5 \mu \mathrm{m}$, Welch Co, Shanghai, China) by an autosampler. Identification and quantification of amino acids was aided with the certified reference standards.

\section{7. $16 \mathrm{~S}$ rRNA Sequencing and Analysis}

Bacterial DNA were extracted from frozen cecal digesta using a QIAamp DNA Stool Mini Kit (QIAGEN, Venlo, The Netherlands) by following the manufacturer's protocol. 16S rRNA paired-end sequencing targeting the V3-V4 hypervariable region was performed in Illumina HiSeq 2500 platform and the gene sequence analysis referred to our previous work [15]. Greengenes database based on the RDP classifier (V2.2) was used on taxonomic classification. 


\subsection{Statistical Analysis}

The data of results were collected and calculated individually for each replicate, and checked for the normality test firstly. Statistical analysis of the results was performed using GraphPad Prism version 8 (GraphPad Software, La Jolla, CA, USA) and R software (V2.16.0). Statistical differences were evaluated by one-way analysis of variance (ANOVA) with Tukey's multiple comparison posttests or by the non-parametric factorial Kruskal-Wallis test. $p$-value $<0.05$ was considered significant ${ }^{*} p<0.05$, $\left.{ }^{* *} p<0.01,{ }^{* * *} p<0.001\right)$ and $0.05<p$-value $<0.10$ was discussed as tendencies.

\section{Results}

\subsection{Dietary GML Improved the Reproductive Performance in Late Laying Period}

As showed in Figure 1a, the laying rate gradually decreased from $96.83 \%$ to $84.42 \%$ and the egg weight (Figure 1b) increased with increasing weeks of age in the CON group. The feed conversion rate (FCR, Figure 1c) increased during 40-52 weeks of age, but showed a sharp decrease at 53-64 weeks of age. The broken rate showed a continuous increase at the end of the trial (Figure 1d). In addition, this plot also showed that dietary GML notably affected the laying rate and feed conversion rate from 52 weeks of age. Moreover, statistical analysis revealed that the laying rate (Figure 1a) of 150 and $300 \mathrm{mg} / \mathrm{kg}$ GML supplementation group increased by $2.42 \%(p=0.017)$ and $2.57 \%(p=0.012)$, respectively, but the FCR (Figure 1c) in GML150 and GML300 group reduced by $2.79 \%(p=0.005)$ and $2.69 \%(p=0.010)$, respectively, compared to the CON group during the whole experimental period.
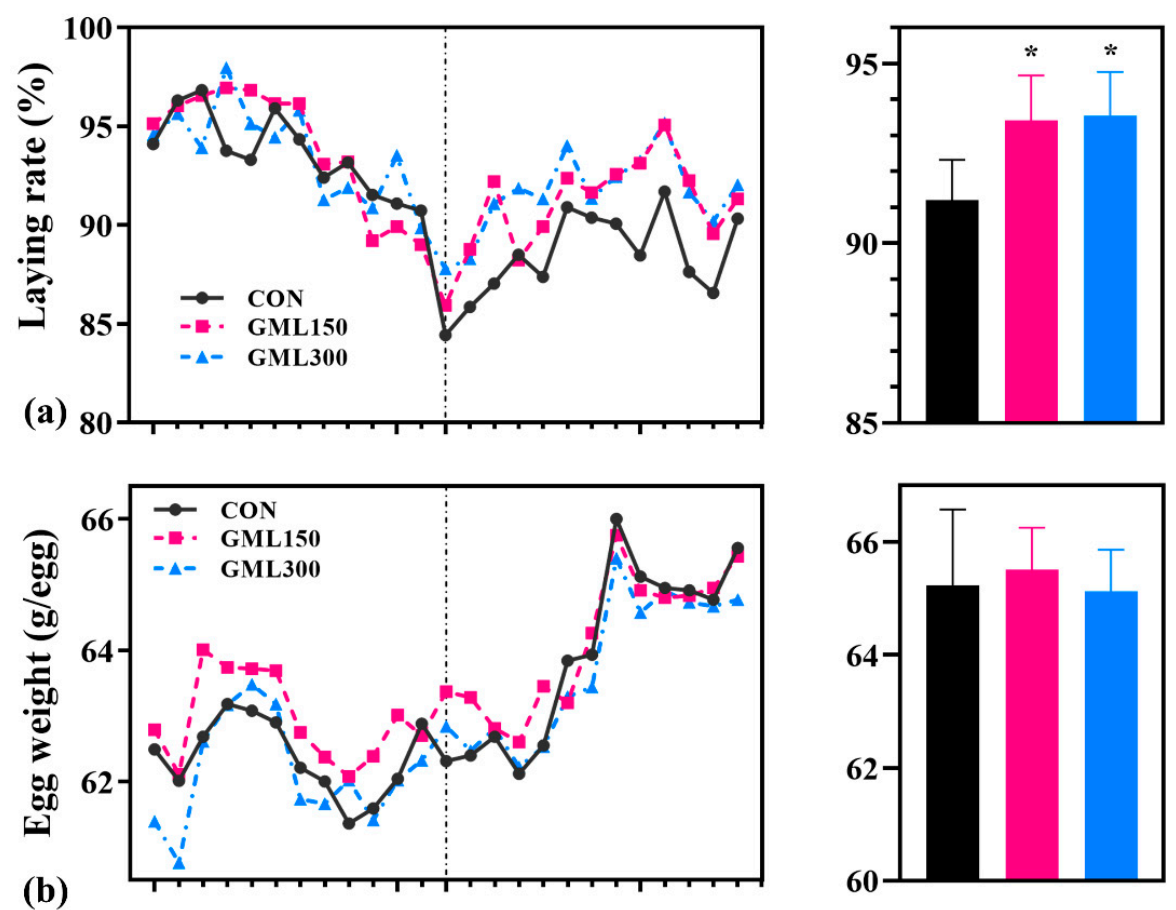

Figure 1. Cont. 

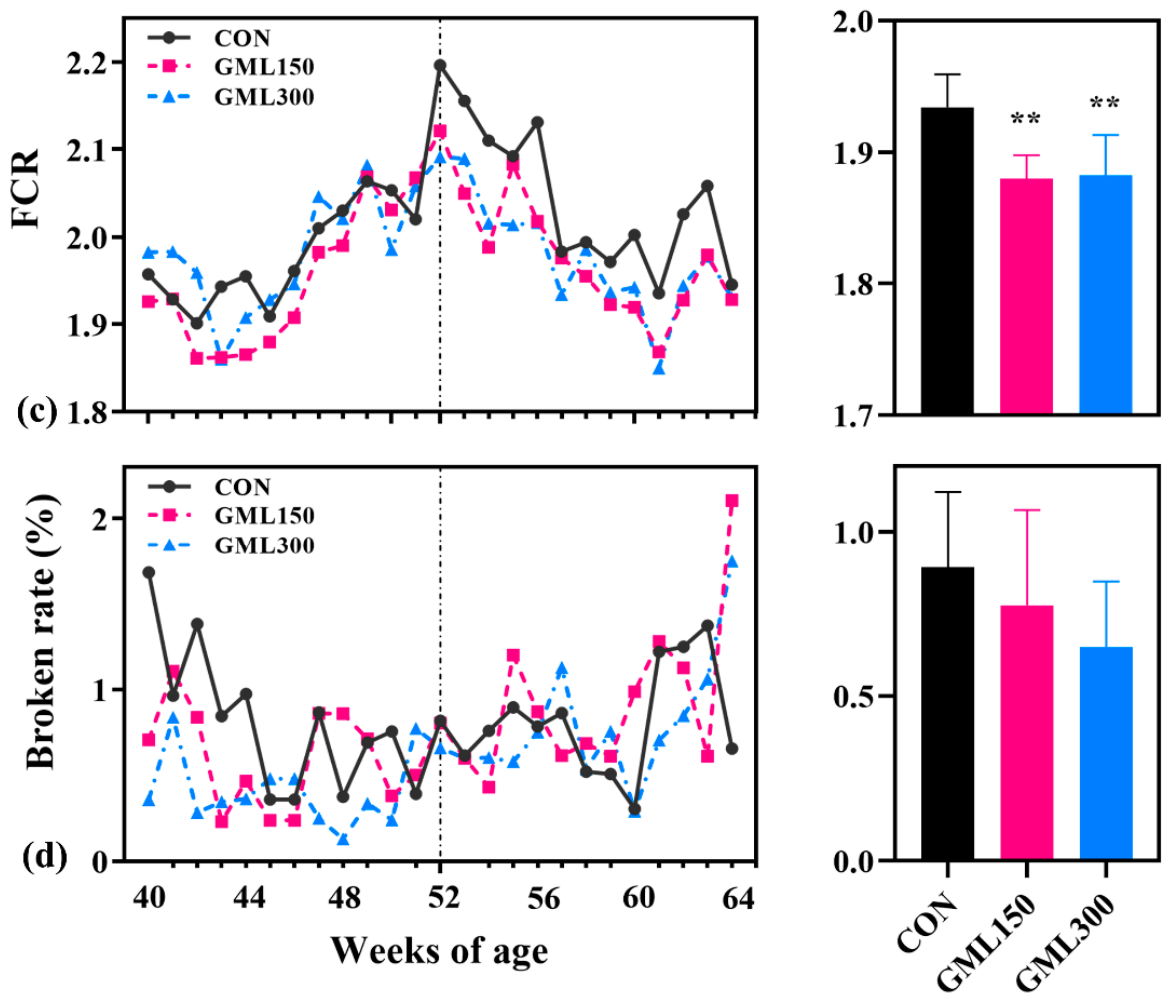

Figure 1. Effect of dietary GML on productive performance during 40-64 weeks of age. (a) Laying rate; (b) egg weight; (c) feed conversion rate (FCR) and (d) broken rate. All data were expressed as means $\pm \operatorname{SD}(n=6)$. Asterisk indicates significantly differences according to ANOVA $(* p<0.05$, ** $p<0.01)$.

\subsection{Dietary GML Improved Egg Quality in the Late Laying Period}

No significant differences on albumen height (Figure 2a) and Haugh units (Figure 2b) were observed, but the eggshell strength at 56 weeks of age (Figure 2c) in the GML150 group and eggshell thickness at 56 and 64 weeks of age (Figure 2d) in GML150 and GML300 group were significantly increased $(p<0.05)$ compared to the CON group. These results indicated that dietary GML improved the eggshell quality of fresh eggs produced at the late laying period.
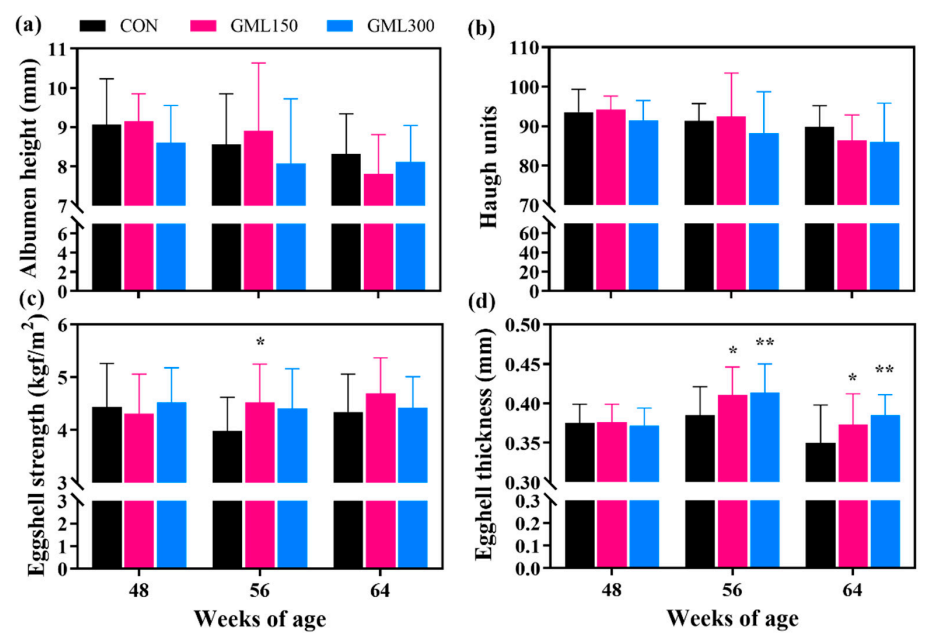

Figure 2. Effect of dietary GML on egg quality of Hy-line brown laying hens. (a) Albumen height; (b) Haugh units; (c) eggshell strength and (d) eggshell thickness. All data were expressed as means \pm SD $(n=6)$. Asterisk indicates significantly differences according to ANOVA $\left({ }^{*} p<0.05,{ }^{* *} p<0.01\right)$. 


\subsection{Dietary GML Affected Serum Parameters}

Significant increased TG, TC and LDL-C level were observed in the GML300 group compared with the CON group, but these indices in the GML150 group showed no significant change (Table 2). Serum Ca and total antioxidant capacity (T-AOC) in the GML300 group were significantly higher than that of the CON group. Serum FSH, LH and E2 level in the GML150 group increased by $25.77 \%, 27.23 \%$ and $18.13 \%$, separately, but no significant differences were observed compared to the CON group. However, significantly increased FSH, LH and E2 content were detected in the GML300 group, and the values were 1.82, 1.57 and 2.12 times higher than that of the CON group, respectively.

Table 2. Effect of dietary GML on serum parameters of late laying period hens.

\begin{tabular}{cccccc}
\hline Parameters & CON & GML150 & GML300 & SEM & $p$ Value \\
\hline TG $(\mathrm{mM})$ & $10.57 \pm 2.17$ & $13.88 \pm 2.88$ & $17.86 \pm 3.16^{* *}$ & 0.77 & 0.001 \\
TC $(\mathrm{mM})$ & $4.96 \pm 0.75$ & $5.194 \pm 0.89$ & $6.78 \pm 0.37 * *$ & 0.22 & 0.001 \\
HDL-C $(\mathrm{mM})$ & $2.69 \pm 0.68$ & $2.74 \pm 0.83$ & $3.23 \pm 0.83$ & 0.12 & 0.122 \\
LDL-C $(\mathrm{mM})$ & $0.74 \pm 0.08$ & $0.88 \pm 0.24$ & $0.89 \pm 0.08^{*}$ & 0.08 & 0.038 \\
Ca $(\mathrm{mM})$ & $1.32 \pm 0.03$ & $1.35 \pm 0.08$ & $1.41 \pm 0.04^{* *}$ & 0.01 & 0.001 \\
AKP $(\mathrm{mM})$ & $26.20 \pm 8.07$ & $27.61 \pm 5.64$ & $26.58 \pm 5.57$ & 1.57 & 0.821 \\
Glucose $(\mathrm{mM})$ & $16.55 \pm 2.91$ & $15.47 \pm 3.87$ & $18.43 \pm 3.33$ & 0.54 & 0.074 \\
Total protein $(\mathrm{g}$ protein/L) & $28.64 \pm 1.86$ & $29.86 \pm 2.31$ & $29.21 \pm 4.43$ & 0.46 & 0.562 \\
T-AOC $(\mathrm{U} / \mathrm{mL})$ & $364.83 \pm 38.44$ & $435.56 \pm 78.60$ & $457.92 \pm 76.39 *$ & 19.01 & 0.008 \\
FSH $(\mathrm{ng} / \mathrm{mL})$ & $32.32 \pm 10.78$ & $40.65 \pm 15.52$ & $91.30 \pm 49.05 * *$ & 12.34 & 0.001 \\
LH $(\mathrm{pg} / \mathrm{mL})$ & $487.95 \pm 124.54$ & $620.84 \pm 163.80$ & $1254.40 \pm 475.56 * *$ & 8.34 & 0.001 \\
E2 $(\mathrm{pg} / \mathrm{mL})$ & $120.39 \pm 19.71$ & $147.58 \pm 57.43$ & $404.29 \pm 288.13 * *$ & 40.68 & 0.001 \\
\hline
\end{tabular}

TG, triglycerides; TC, total cholesterol; HDL-C, high-density lipoprotein cholesterol; LDL-C, low-density lipoprotein cholesterol; $\mathrm{Ca}$, calcium; AKP, alkaline phosphatase; T-AOC, total antioxidant capacity; $\mathrm{FSH}$, follicle stimulating hormone; LH, luteinizing hormone; E2, estradiol. All data were expressed as means $\pm \mathrm{SD}(n=18)$. Asterisk indicates significantly differences according to ANOVA $\left({ }^{*} p<0.05,{ }^{* *} p<0.01,{ }^{* * *} p<0.001\right)$.

\subsection{Effect of Dietary GML on the Yolk Fatty Acids Profile}

There was no effect of dietary GML on yolk fatty acids compositions was found (Table S1).

\subsection{Effect of Dietary GML on the Albumen Amino Acids Profile}

The content of aspartic acid and total flavor amino acids (FAA) in both GML150 and GML300 group increased significantly compared to the CON group (Table 3). Compared with the CON group, the FAA content in $150 \mathrm{mg} / \mathrm{kg}$ and $300 \mathrm{mg} / \mathrm{kg}$ GML supplementation groups increased by $25.87 \%$ $(p=0.011)$ and $19.18 \%(p=0.046)$, respectively, and the total amino acids (TAA) increased by 11.68 $(p=0.046)$ and $5.27 \%$ relative to that of the CON group. These data demonstrated that dietary GML altered albumen amino acids composition, especially in the content of FAA in late laying period hens.

Table 3. Effect of dietary GML on albumen amino acids profile of late laying period hens.

\begin{tabular}{cccccc}
\hline g/100 g & CON & GML150 & GML300 & SEM & $p$ Value \\
\hline \multicolumn{7}{c}{ Umami taste amino acids } \\
Aspartic acid & $5.32 \pm 1.47$ & $7.58 \pm 0.58^{*}$ & $7.44 \pm 0.87 *$ & 0.41 & 0.018 \\
Glutamic acid & $4.83 \pm 1.33$ & $5.86 \pm 0.66$ & $5.45 \pm 0.85$ & 0.27 & 0.286 \\
\multicolumn{7}{c}{ Sweet taste amino acids } & & & \\
Serine & $3.50 \pm 0.66$ & $3.88 \pm 0.40$ & $3.29 \pm 0.35$ & 0.14 & 0.247 \\
Glycine & $2.80 \pm 0.33$ & $3.04 \pm 0.21$ & $2.87 \pm 0.19$ & 0.07 & 0.344 \\
Alanine & $5.23 \pm 0.66$ & $5.87 \pm 0.55$ & $5.69 \pm 0.10$ & 0.15 & 0.166 \\
$\Sigma$ FAA & $20.80 \pm 3.06$ & $26.18 \pm 2.00 *$ & $24.79 \pm 2.95 *$ & 0.85 & 0.014 \\
\hline
\end{tabular}


Table 3. Cont.

\begin{tabular}{cccccc}
\hline g/100 $\mathrm{g}$ & CON & GML150 & GML300 & SEM & $p$ Value \\
\hline EAA & & & & & \\
Lysine & $5.44 \pm 1.21$ & $6.08 \pm 0.84$ & $4.89 \pm 0.29$ & 0.25 & 0.198 \\
Methionine & $3.22 \pm 0.43$ & $3.39 \pm 0.19$ & $3.22 \pm 0.20$ & 0.08 & 0.615 \\
Threonine & $2.72 \pm 0.43$ & $2.80 \pm 0.64$ & $2.72 \pm 0.56$ & 0.13 & 0.955 \\
Leucine & $8.29 \pm 0.80$ & $8.83 \pm 0.66$ & $8.50 \pm 0.44$ & 0.17 & 0.441 \\
Isoleucine & $5.16 \pm 0.57$ & $5.53 \pm 0.51$ & $5.35 \pm 0.40$ & 0.13 & 0.514 \\
Arginine & $3.86 \pm 0.97$ & $4.58 \pm 0.47$ & $4.37 \pm 0.48$ & 0.19 & 0.270 \\
Valine & $5.86 \pm 0.96$ & $6.70 \pm 0.86$ & $6.70 \pm 0.34$ & 0.22 & 0.190 \\
Phenylalanine & $5.30 \pm 0.54$ & $5.58 \pm 0.42$ & $5.44 \pm 0.43$ & 0.12 & 0.646 \\
Histidine & $1.58 \pm 0.23$ & $1.67 \pm 0.19$ & $1.60 \pm 0.23$ & 0.05 & 0.784 \\
$\Sigma$ EAA & $41.43 \pm 5.72$ & $43.41 \pm 5.41$ & $42.05 \pm 3.82$ & 1.62 & 0.794 \\
Proline & $5.33 \pm 0.69$ & $5.50 \pm 0.52$ & $5.02 \pm 0.33$ & 0.14 & 0.460 \\
Tyrosine & $2.90 \pm 0.50$ & $3.07 \pm 0.07$ & $2.79 \pm 0.59$ & 0.11 & 0.680 \\
Cysteine & $0.71 \pm 0.87$ & $1.99 \pm 1.64$ & $1.40 \pm 0.94$ & 0.39 & 0.476 \\
$\Sigma$ AA & $72.42 \pm 6.07$ & $80.88 \pm 5.44 *$ & $76.24 \pm 4.32$ & 2.18 & 0.074 \\
\hline
\end{tabular}

$\overline{\Sigma \text { FAA, the sum of flavor amino acids (sweet and umami taste amino acids); } \Sigma \text { EAA, the sum of essential amino }}$ acids; $\Sigma$ AA, the sum of total amino acids. All data were expressed as means $\pm \operatorname{SD}(n=6)$. Asterisk indicates significantly differences according to ANOVA $\left({ }^{*} p<0.05\right)$.

\subsection{Dietary GML Altered the Gut Microbiota in Late Laying Period Hens}

The Venn diagrams showed that a total of 9075 OTUs was obtained in all groups, and 802, 1187 and 886 specific operational taxonomic units (OTUs) existed in the CON, GML150 and GML300 group, respectively (Figure 3a). The $\alpha$-diversity was reflected by Chao and ACE (bacterial richness), Shannon and Simpson indices (bacterial diversity and evenness) calculated based on OTUs. The Shannon, Simpson and Chao indices showed no changes, but the ACE was higher in the GML300 group, suggesting that the bacterial richness of gut microbiota was increased by $300 \mathrm{mg} / \mathrm{kg}$ GML supplementation (Figure 3b). The principal component analysis (PCA) plot demonstrated that the data points of GML-treated groups shifted away from the CON group, indicating that dietary GML changed the gut microbiota community in a characteristic direction (Figure 3c). Statistical analysis revealed that the GML300 group differed from the CON group on PC1 $(36.71 \%, p=0.006)$, and the GML150 group was different from the CON group on PC2 $(14.30 \%, p<0.001)$ calculated by Anoism PCA distance (Figure 3c). Taxonomic profiling showed a diverse gut microbiota community in CON and GML-treated hens at the phylum level (Figure 3d). Inclusion of $300 \mathrm{mg} / \mathrm{kg}$ GML in diet significantly increased the relative abundance of phyla Fusobacteria and Deferribacteres compared with the CON group (Figure 3e). At the genus level (Table 4), $300 \mathrm{mg} / \mathrm{kg}$ GML supplementation significantly increased the abundance of genera Fusobacterium, Mucispirillum and norank_f_Pedosphaeraceae, and $150 \mathrm{mg} / \mathrm{kg}$ GML supplementation significantly increased the abundance of genera Rikenellaceae_RC9_gut_group

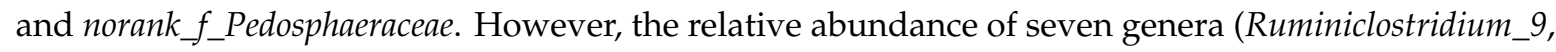
Megasphaera, Negativibacillus, Ruminococcaceae_UCG-009, Family_XIII_AD3011_group, Angelakisella and Eubacterium_nodatum_group) in the GML300 group and five genera (Ruminiclostridium_9, norank_f_Erysipelotrichaceae, GCA-900066225, Angelakisella and Eubacterium_nodatum_group) in the GML150 group belonging to the phylum Firmicutes were all decreased. 
(a)

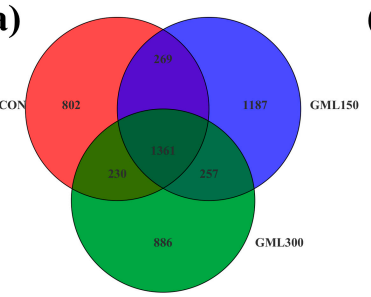

(c)

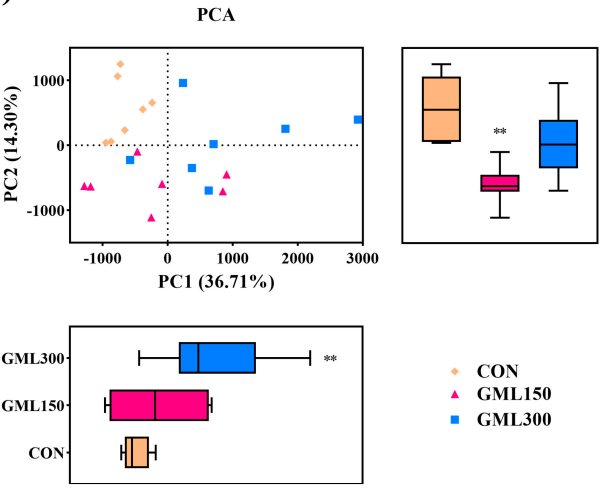

(b)

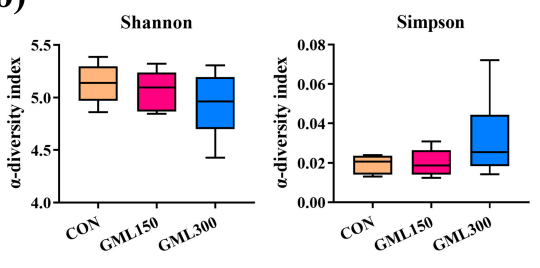

(d)

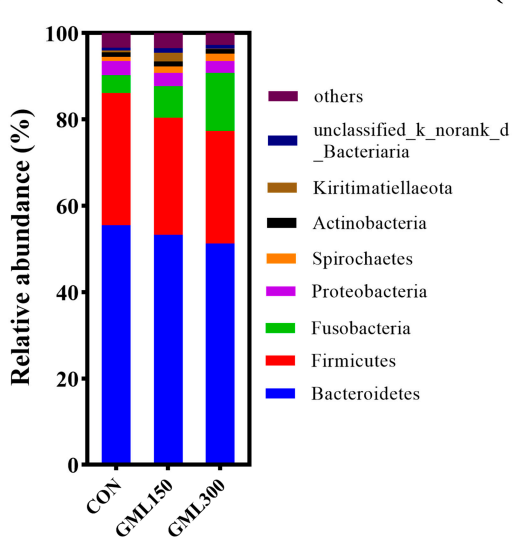

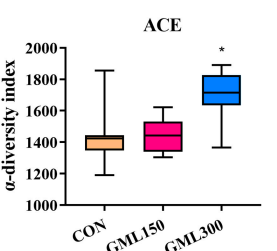

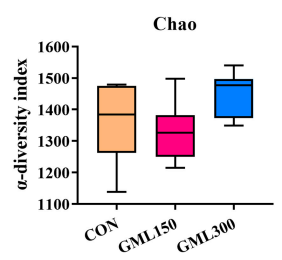

(e)
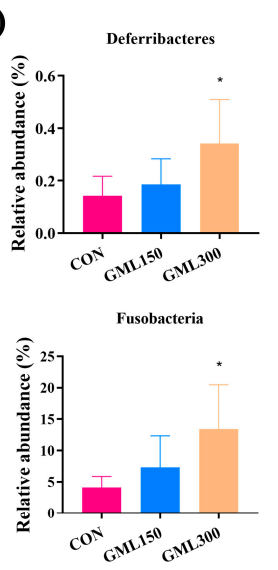

Figure 3. GML supplementation altered the gut microbiota community in hens. (a) A Venn diagram showing operational taxonomic units (OTUs) differences among treatments. (b) $\alpha$-diversity: Chao, ACE, Shannon and Simpson. (c) $\beta$-diversity: principal component analysis (PCA). (d) Phylum level among different groups. (e) Differential phyla detected among different groups. Data are expressed as means $\pm \mathrm{SD}(n=7)$. Asterisk indicates significantly differences according to the Kruskal-Wallis test $\left({ }^{*} p<0.05, * * p<0.01\right)$.

Table 4. GML altered the gut microbiota community in hens at the genus level.

\begin{tabular}{|c|c|c|c|c|c|}
\hline \multirow{2}{*}{ Genus } & \multicolumn{5}{|c|}{ Relative Abundance (\%) } \\
\hline & CON & GML150 & GML300 & SEM & $p$ Value \\
\hline p_Bacteroidetes & & & & & \\
\hline $\begin{array}{c}\text { Rikenellaceae_RC9_gut_group } \\
\text { p_Fusobacteria }\end{array}$ & $8.035 \pm 3.252$ & $12.690 \pm 4.134^{*}$ & $9.991 \pm 3.346$ & 0.859 & 0.078 \\
\hline $\begin{array}{l}\text { Fusobacterium } \\
\text { p_Firmicutes }\end{array}$ & $4.124 \pm 1.759$ & $7.332 \pm 5.010$ & $13.430 \pm 7.030$ * & 1.362 & 0.010 \\
\hline Ruminiclostridium_9 & $1.314 \pm 0.405$ & $0.898 \pm 0.219 *$ & $0.887 \pm 0.174 *$ & 0.074 & 0.017 \\
\hline Megasphaera & $0.347 \pm 0.238$ & $0.282 \pm 0.554$ & $0.075 \pm 0.084 *$ & 0.077 & 0.048 \\
\hline Negativibacillus & $0.103 \pm 0.053$ & $0.121 \pm 0.051$ & $0.049 \pm 0.031 * *$ & 0.012 & 0.031 \\
\hline norank_f_Erysipelotrichaceae & $0.082 \pm 0.047$ & $0.024 \pm 0.023 *$ & $0.062 \pm 0.047$ & 0.010 & 0.031 \\
\hline GCA-900066225 & $0.057 \pm 0.033$ & $0.025 \pm 0.019 *$ & $0.058 \pm 0.028$ & 0.007 & 0.012 \\
\hline Ruminococcaceae_UCG-009 & $0.057 \pm 0.023$ & $0.046 \pm 0.0 .11$ & $0.032 \pm 0.011 *$ & 0.004 & 0.012 \\
\hline Family_XIII_AD3011_group & $0.056 \pm 0.029$ & $0.037 \pm 0.016$ & $0.027 \pm 0.016 *$ & 0.005 & 0.061 \\
\hline Angelakisella & $0.053 \pm 0.019$ & $0.033 \pm 0.014 *$ & $0.030 \pm 0.010 *$ & 0.004 & 0.046 \\
\hline Eubacterium_nodatum_group & $0.043 \pm 0.007$ & $0.024 \pm 0.016^{*}$ & $0.019 \pm 0.010^{* * *}$ & 0.003 & 0.006 \\
\hline $\begin{array}{l}\text { P_Deferribacteres } \\
\text { Mucispirillum }\end{array}$ & $0.142 \pm 0.075$ & $0.185 \pm 0.099$ & $0.343 \pm 0.167 *$ & 0.031 & 0.043 \\
\hline p_Verrucomicrobia & & & & & \\
\hline norank_f_Pedosphaeraceae & $0.043 \pm 0.021$ & $0.104 \pm 0.059 *$ & $0.085 \pm 0.038 *$ & 0.010 & 0.036 \\
\hline
\end{tabular}

Data are expressed as means $\pm \mathrm{SD}(n=7)$. Asterisk indicates significantly differences according to the Kruskal-Wallis test $\left.{ }^{*} p<0.05,{ }^{* *} p<0.01,{ }^{* * *} p<0.001\right)$. 


\section{Discussion}

In the current study, laying hens fed diets containing 150 and $300 \mathrm{mg} / \mathrm{kg}$ GML showed a significant increased laying rate and reduced FCR during 40-64 weeks of age, which was consistent with our previous study, where $150-450 \mathrm{mg} / \mathrm{kg}$ GML exerted beneficial impact on laying performance and feed efficiency in hens during 44-52 weeks of age [16]. Moreover, we found that it was during the later late laying period (52-64 weeks of age) that GML improved the laying performance, feed efficiency and egg quality after 24 weeks of nutrition intervention, though the improvements of the laying performance were also observed during the 40-52 weeks of age. There were growing evidences documented that dietary free and esterified medium chain fatty acids (MCFAS) was beneficial for poultry production and feed nutrients utilization [12]. According to Wang and Kim [20], dietary caprylic acid improved average egg weight and feed efficiency in Hy-Line brown, and had positive effects on serum blood metabolism and health status. Dietary GML supplementation increased body weight, feed consumption and carcass yield, and decreased the FCR in the broiler, which were also better for the health of chicken [13,14]. Dietary fat, rich in free lauric acids improved the FCR and breast muscle yield in broilers [21]. Laying performance was affected by many factors such as genetics, management, feed formulation and environment. However, during the late laying period, the decline of egg production was mainly due to the decreased yolk synthesis and accumulation by lowered sex hormone concentrations $[1,3,4]$. FSH is the main hormone regulating the development and maturation of small follicles in hens, while LH mainly promotes the secretion of progesterone, and E2 can promote follicular development via feedback effects on the hypothalamus and pituitary. Thus, the serum sex hormones including FSH, LH and E2 have been considered as a sensitive indicator for laying performance in aged hens. Application of some feed additives and chemicals such as antibiotics, somatotropin and preservatives in feed can enhance the laying rate by increasing the sex hormone level [1]. As demonstrated by Zhang et al. [22], dietary $\gamma$-aminobutyric acid significantly increased laying performance by increasing concentrations of serum FSH, LH and other hormones. In the present study, the increased serum FSH, LH and E2 level induced by GML supplementation were responsible for the improvement of productive performance. As similar results were published in our previous study, where dietary GML improved the laying rate via stimulating the secretion of FSH and LH in hens [16].

During the late laying period, egg quality is rapidly declined along with a higher cracked rate, increased egg size, thinner eggshell, lower albumen height, shortened storage time and poor flavor [5,23]. Eggshell thickness and strength were an essential attribute for egg production [24], and the improvement of eggshell quality was of great importance for late laying period hens. In the current study, the egg weight and broken rate of the CON group were continuously increased during the end of the experimental period, suggesting that the egg quality was decreased as hens aged (Figure 1). However, the eggshell strength and eggshell thickness were notably improved in the GML150 and GML300 group compared with the CON group. These changes may be derived from the addition of GML, as MCFAs were reported to be able to participate in animal calcium metabolism [25]. This was also supported by the increased serum calcium concentration in the GML150 (1.32 vs. $1.35 \mathrm{mM}$ ) and GML300 (1.32 vs. $1.41 \mathrm{mM}, p<0.05$ ) group. Similar results were reported by Świątkiewicz [26], who demonstrated that dietary MCFAs notably enhanced both eggshell density and eggshell breaking strength in laying hens.

Egg proteins are easily digestible and rich in all the essential amino acids (EAA) for the human body, and eggs are suitable for people at all ages [22]. Egg proteins are made of amino acids and the concentration of total amino acids (TAA), EAA and flavor amino acids (FAA) play an important role on egg nutritive value and flavor. Therefore, the albumen amino acids profile could influence both the nutritive values and flavor of the eggs [27]. In the current study, no significant differences of EAA were observed among groups, but the total FAA content in the GML supplementation groups was significantly increased. Moreover, the relative contents of FAA detected in the present study accounted for approximately 32.37\% (GML150 group) and 32.52\% (GML300 group) in the TAA content. 
The desirable amino acids possessing an umami (Glu and Asp), or sweet (Ser, Gly and Ala) taste [28,29]. Despite the fact that flavor amino acids contributing to basic tastes (sweet and umami), they are also flavor precursor substances when cooking and play an important role in flavor formation [30-32]. Therefore, the notable changes in these amino acids could inevitably lead to the alteration of flavor for the eggs produced in hens fed with GML supplementation. Additionally, the TAA in 150 and $300 \mathrm{mg} / \mathrm{kg}$ GML supplementation group were also notably increased, indicating that dietary GML also increased the nutritive values of eggs. These effects of GML on egg albumen composition may derive from the impact of GML on amino acids metabolism in laying hens induced by decreased FCR, but warranted further study. Consequently, the improvements in albumen amino acids profile by GML supplementation could result in better flavor and nutritional benefits.

The serum cholesterol (TC), triglycerides (TG) and low-density lipoprotein cholesterol (LDL-C) content were significantly increased in the GML300 group compared to the CON group, suggesting that relative high dose of GML supplementation affected blood lipid metabolism in late laying period hens. Conversely, these results were not consistent with our previous study, where inclusion of $150-450 \mathrm{mg} / \mathrm{kg}$ GML in diet reduced the concentration of serum TC, TG and LDL-C, but increased the HDL-C content in hens [16]. Results obtained in the application of MCFAs in broiler chickens also showed that the LDL-C concentration was decreased and the HDL-C was increased in the treated groups [33]. Interestingly, the yolk fatty acids composition in the present study showed no differences in GML supplementation groups, and these results were also contrary to our previous study [16]. The discrepancy may be due to the differences on the ages of hens, feed formulation, environment, management and the form of MCFAs. Generally, the effect of dietary GML on serum lipid metabolism had not reached consensus, which deserved further analysis.

The gut microbiota of the chicken plays an important role in prevention of infectious diseases, regulation of nutrient digestion and metabolism, and immune homeostasis of the host [34]. In the present study, alpha and beta diversity indicators revealed that the gut microbiota composition and richness in the GML supplementation groups differed from the CON group. Indeed, the abundance of phyla Fusobacteria (genus Fusobacterium) and Deferribacteres (genus Mucispirillum) was significantly increased in the GML300 groups, while the phyla Firmicutes (Ruminiclostridium_9, Megasphaera, Negativibacillus, Ruminococcaceae_UCG-009, Family_XIII_AD3011_group, Angelakisella and Eubacterium_nodatum_group) was notably decreased. Additionally, the abundance of phyla Firmicutes (Ruminiclostridium_9, norank_f_Erysipelotrichaceae, GCA-900066225, Angelakisella and Eubacterium_nodatum_group) in the GML150 group was reduced. Fusobacterium and its members can act as proinflammatory factors that promote intestinal tumorigenesis, and they were reported to be associated with multifactorial diseases in human [35]. However, it has not been concluded to whether Fusobacterium was the cause of poor laying performance and feed efficiency in laying hens. Kollarcikova et al. considered that a higher level of Fusobacterium was quite undesirable in the cecum of hens because it is not a common gut microbiota member of chickens [36]. A previous study demonstrated that the members of Fusobacterium had no relationship with increased mortality, reduced feed efficiency and decreased egg production and quality of hens under heat stress [37]. Interestingly, high abundance of Fusobacteria and Deferribacteres microbiota was found to be helpful for geese raised in intensive systems to digest the high-grain diet [38]. However, the increase of Fusobacteria by GML supplementation did not have obvious adverse effect on laying performance and feed efficiency in the current study. In addition, dietary GML supplementation in hens decreased some genera of Firmicutes, which was linked to the efficiency of nutrients absorption, and consequently to energy harvest from the diet [39]. Many members of Firmicutes could encode carbohydrate-active enzymes and increase carbohydrates hydrolysis and utilization [40], suggesting that the carbohydrate metabolism might be influenced by dietary GML supplementation. GML had the strongest antimicrobial capacity among the medium chain 1-monoglycerides (MGs) and possess a broad antibacterial spectrum, especially in suppressing the growth of gram-positive bacteria in vitro [11]. Dietary GML in the diet of mice significantly altered the composition, microbial metabolite and function of gut microbiota, which 
consequently affected the animal performance and nutrients utilization [41,42]. The present study revealed that the gut microbiota composition and function were altered by GML supplementation, but their relationship with laying performance and feed efficiency required further study.

\section{Conclusions}

A significant improvement in reproductive performance (laying rate and FCR) in GML-containing groups was observed in our study during 40-64 weeks of age, based on notable increases in serum sex hormone levels (FSH, LH and E2). Moreover, the egg quality (eggshell thickness and strength) and albumen amino acids profile (FAA and TAA) were also significantly improved by GML supplementation at the later late laying period. These findings provided us with a new approach to simultaneously improve egg production and enhance the egg quality at aged hens. In addition, GML supplementation in the diet of laying hens altered the structure and function of gut microbiota, though their effect on laying performance needed further analysis. Considering the laying rate, FCR, egg quality, albumen amino acids, serum indices and gut microbiota, a supplementation levels of $150 \mathrm{mg} / \mathrm{kg}$ GML was recommended in the late laying period Hy-Line Brown hens.

Supplementary Materials: The following are available online at http://www.mdpi.com/2077-0472/10/7/250/s1, Table S1: Effect of dietary GML on yolk fatty acids composition of late laying period hens.

Author Contributions: T.L. and F.F. designed research, T.L. and C.L. performed the experiment, data collection and article writing. Y.L. and F.F. revised the article. All authors have read and agreed to the published version of the manuscript.

Funding: This research was funded by Technology and Achievement Transformation Project of Hangzhou, China (20161631E01), Zhejiang University New Rural Development Research Institute Agricultural Technology Promotion Fund (2017006), Natural Science Foundation of Zhejiang Province (LY18C200006) and Natural Science Foundation of China (31601561).

Acknowledgments: We're especially grateful for Yanyun Ying, Tingting Bu, Jianli Wang, Hangzhou Guo and all people participating in the preparation and analysis of this study.

Conflicts of Interest: The authors declare that they have no conflict of interest.

\section{References}

1. Liu, Y.; Li, Y.; Liu, H.N.; Suo, Y.L.; Hu, L.L.; Feng, X.A.; Zhang, L.; Jin, F. Effect of quercetin on performance and egg quality during the late laying period of hens. Br. Poult. Sci. 2013, 54, 510-514. [CrossRef] [PubMed]

2. Joyner, C.J.; Peddie, M.J.; Taylor, T.G. The effect of age on egg production in the domestic hen. Gen. Comp. Endocrinol. 1987, 65, 331-336. [CrossRef]

3. Liu, H.K.; Long, D.W.; Bacon, W.L. Preovulatory luteinizing hormone surge interval in old and young laying turkey hens early in the egg production period. Poult. Sci. 2001, 80, 1364-1370. [CrossRef] [PubMed]

4. Xie, T.; Bai, S.P.; Zhang, K.Y.; Ding, X.M.; Wang, J.P.; Zeng, Q.F.; Peng, H.W.; Lu, H.Y.; Bai, J.; Xuan, Y.; et al. Effects of Lonicera confusa and Astragali Radix extracts supplementation on egg production performance, egg quality, sensory evaluation, and antioxidative parameters of laying hens during the late laying period. Poult. Sci. 2019, 98, 4838-4847. [CrossRef]

5. Liu, Z.; Sun, C.J.; Yan, Y.Y.; Li, G.Q.; Shi, F.Y.; Wu, G.Q.; Liu, A.Q.; Yang, N. Genetic variations for egg quality of chickens at late laying period revealed by genome-wide association study. Sci. Rep. 2018, 8, 11. [CrossRef]

6. Wen, Z.; Wu, Y.; Qi, Z.; Li, X.; Li, F.; Wu, X.; Yang, P. Rubber seed oil supplementation enriches n-3 polyunsaturated fatty acids and reduces cholesterol contents of egg yolks in laying hens. Food Chem. 2019, 301, 125198. [CrossRef]

7. Abdel-Wareth, A.A.A.; Lohakare, J.D. Effect of dietary supplementation of peppermint on performance, egg quality, and serum metabolic profile of Hy-Line Brown hens during the late laying period. Anim. Feed Sci. Technol. 2014, 197, 114-120. [CrossRef]

8. Wang, X.C.; Wang, X.H.; Wang, J.; Wang, H.; Zhang, H.J.; Wu, S.G.; Qi, G.H. Dietary tea polyphenol supplementation improved egg production performance, albumen quality, and magnum morphology of Hy-Line Brown hens during the late laying period. J. Anim. Sci. 2018, 96, 225-235. [CrossRef] 
9. Baltic, B.; Starcevic, M.; Dordevic, J.; Mrdovic, B.; Markovic, R. Importance of medium chain fatty acids in animal nutrition. IOP Conf. Ser. Earth Environ. Sci. 2017, 85, 012048. [CrossRef]

10. Jiang, Z.; Zhao, M.; Zhang, H.; Li, Y.; Liu, M.; Feng, F. Antimicrobial emulsifier-Glycerol monolaurate induces metabolic syndrome, gut microbiota dysbiosis and systemic low-grade inflammation in low-fat diet fed mice. Mol. Nutr. Food Res. 2018, 63, 1700547. [CrossRef]

11. Batovska, D.I.; Todorova, T.; Tsvetkova, V.; Najdenski, H.M. Antibacterial study of the medium chain fatty acids and their 1-monoglycerides: Individual effects and synergistic relationships. Pol. J. Microbiol. 2009, 58, 43-47. [PubMed]

12. van der Aar, P.J.; Molist, F.; van der Klis, J.D. The central role of intestinal health on the effect of feed additives on feed intake in swine and poultry. Anim. Feed Sci. Technol. 2017, 233, 64-75. [CrossRef]

13. Fortuoso, B.F.; dos Reis, J.H.; Gebert, R.R.; Barreta, M.; Griss, L.G.; Casagrande, R.A.; de Cristo, T.G.; Santiani, F.; Campigotto, G.; Rampazzo, L.; et al. Glycerol monolaurate in the diet of broiler chickens replacing conventional antimicrobials: Impact on health, performance and meat quality. Microb. Pathog. 2019, 129, 161-167. [CrossRef]

14. Mustafa, N.G. Biochemical trails associated with different doses of alpha-monolaurin in chicks. Adv. Anim. Vet. Sci. 2019, 7, 187-192. [CrossRef]

15. Zhao, M.J.; Cai, H.Y.; Liu, M.Y.; Deng, L.L.; Li, Y.; Zhang, H.; Feng, F.Q. Dietary glycerol monolaurate supplementation for the modification of functional properties of egg white protein. J. Sci. Food Agric. 2019, 99, 3852-3859. [CrossRef] [PubMed]

16. Liu, M.; Wang, J.; Feng, F. Effects of glycerol monolaurate on production performance, egg quality, serum parameters, immune organ index and abdominal fat morphology of laying hens. China Poult. 2017, 39, $24-30$.

17. National Research Council, Nutrient Requirements of Poultry, 10th ed.; National Academies Press: Washington, DC, USA, 2017.

18. Mrf, L.; Jks, T.; Kim, E.J.; Scollan, N.D. Beef, chicken and lamb fatty acid analysis-A simplified direct bimethylation procedure using freeze-dried material. Meat Sci. 2012, 92, 863-866.

19. Gheshlaghi, R.; Scharer, J.M.; Moo-Young, M.; Douglas, P.L. Application of statistical design for the optimization of amino acid separation by reverse-phase HPLC. Anal Biochem. 2008, 383, 93-102. [CrossRef]

20. Wang, J.P.; Kim, I.H. Effect of caprylic acid and Yucca schidigera extract on production performance, egg quality, blood characteristics, and excreta microflora in laying hens. Br. Poult. Sci. 2011, 52, 711-717. [CrossRef]

21. Zeitz, J.O.; Fennhoff, J.; Kluge, H.; Stangl, G.I.; Eder, K. Effects of dietary fats rich in lauric and myristic acid on performance, intestinal morphology, gut microbes, and meat quality in broilers. Poult. Sci. 2015, 94, 2404-2413. [CrossRef]

22. Zhang, M.; Zou, X.T.; Hui, L.I.; Dong, X.Y.; Zhao, W. Effect of dietary $\gamma$-aminobutyric acid on laying performance, egg quality, immune activity and endocrine hormone in heat-stressed Roman hens. Anim. Sci. J. 2012, 83, 141-147. [CrossRef] [PubMed]

23. Kim, C.H.; Song, J.H.; Lee, J.C.; Lee, K.W. Age-related changes in egg quality of Hy-Line brown hens. Int. J. Poult. Sci. 2014, 13, 510-514. [CrossRef]

24. Mertens, K.; Bamelis, F.; Kemps, B.; Kamers, B.; Verhoelst, E.; De Ketelaere, B.; Bain, M.; Decuypere, E.; De Baerdemaeker, J. Monitoring of eggshell breakage and eggshell strength in different production chains of consumption eggs. Poult. Sci. 2006, 85, 1670-1677. [CrossRef] [PubMed]

25. Wauquier, F.; Leotoing, L.; Philippe, C.; Spilmont, M.; Coxam, V.; Wittrant, Y. Pros and cons of fatty acids in bone biology. Prog. Lipid Res. 2015, 58, 121-145. [CrossRef]

26. Świątkiewicz, S.; Koreleski, J.; Arczewska, A. Laying performance and eggshell quality in laying hens fed diets supplemented with prebiotics and organic acids. Czech J. Anim. Sci. 2010, 55, 294-306. [CrossRef]

27. Jiang, W.D.; Wu, P.; Tang, R.J.; Liu, Y.; Kuang, S.Y.; Jiang, J.; Tang, L.; Tang, W.N.; Zhang, Y.A.; Zhou, X.Q. Nutritive values, flavor amino acids, healthcare fatty acids and flesh quality improved by manganese referring to up-regulating the antioxidant capacity and signaling molecules TOR and Nrf2 in the muscle of fish. Food Res. Int. 2016, 89, 670-680. [CrossRef]

28. Bermúdez, R.; Franco, D.; Carballo, J.; Sentandreu, M.Á.; Lorenzo, J.M. Influence of muscle type on the evolution of free amino acids and sarcoplasmic and myofibrillar proteins through the manufacturing process of Celta dry-cured ham. Food Res. Int. 2014, 56, 226-235. [CrossRef] 
29. Zhou, C.Y.; Wang, Y.; Cao, J.X.; Chen, Y.J.; Liu, Y.; Sun, Y.Y.; Pan, D.D.; Ou, C.R. The effect of dry-cured salt contents on accumulation of non-volatile compounds during dry-cured goose processing. Poult. Sci. 2016, 95, 2160-2166. [CrossRef]

30. Xiao, Z.; Ge, C.; Zhou, G.; Zhang, W.; Liao, G. 1H NMR-based metabolic characterization of Chinese Wuding chicken meat. Food Chem. 2019, 274, 574-582. [CrossRef]

31. Khan, M.I.; Jo, C.; Tariq, M.R. Meat flavor precursors and factors influencing flavor precursors-A systematic review. Meat Sci. 2015, 110, 278-284. [CrossRef]

32. Liu, C.; Pan, D.; Ye, Y.; Cao, J. 1H NMR and multivariate data analysis of the relationship between the age and quality of duck meat. Food Chem. 2013, 141, 1281-1286. [CrossRef] [PubMed]

33. Shokrollahi, B.; Yavari, Z.; Kordestani, A. Effects of dietary medium-chain fatty acids on performance, carcass characteristics, and some serum parameters of broiler chickens. Br. Poult. Sci. 2014, 55, 662-667. [CrossRef] [PubMed]

34. Pan, D.; Yu, Z. Intestinal microbiome of poultry and its interaction with host and diet. Gut Microbes 2013, 5, 108-119. [CrossRef] [PubMed]

35. Matsukawa, Y.; Kitamura, N.; Kaneko, M.; Yoshioka, D.; Miki, T.; Nishlnarita, S.; Horie, T.; Hosokawa, N.; Iwasaki, Y.; Kumasaka, K. Multibacterial Sepsis in an Alcohol Abuser with Hepatic Cirrhosis. Intern. Med. 2003, 42, 208-210. [CrossRef] [PubMed]

36. Kollarcikova, M.; Kubasova, T.; Karasova, D.; Crhanova, M.; Cejkova, D.; Sisak, F.; Rychlik, I. Use of 16S rRNA gene sequencing for prediction of new opportunistic pathogens in chicken ileal and cecal microbiota. Poult. Sci. 2019, 98, 2347-2353. [CrossRef]

37. Zhu, L.; Liao, R.; Wu, N.; Zhu, G.; Yang, C. Heat stress mediates changes in fecal microbiome and functional pathways of laying hens. Appl. Microbiol. Biotechnol. 2019, 103, 461-472. [CrossRef]

38. Xu, Q.; Yuan, X.; Gu, T.; Li, Y.; Dai, W.; Shen, X.; Song, Y.; Zhang, Y.; Zhao, W.; Chang, G. Comparative characterization of bacterial communities in geese fed all-grass or high-grain diets. PLoS ONE 2017, 12, e0185590. [CrossRef]

39. Zhou, B.-H.; Jia, L.-S.; Wei, S.-S.; Ding, H.-Y.; Yang, J.-Y.; Wang, H.-W. Effects of Eimeria tenella infection on the barrier damage and microbiota diversity of chicken cecum. Poult. Sci. 2020,99, 1297-1305. [CrossRef]

40. Zhang, Y.; Liu, Y.; Li, J.; Xing, T.; Jiang, Y.; Zhang, L.; Gao, F. Dietary resistant starch modifies the composition and function of caecal microbiota of broilers. J. Sci. Food Agric. 2020, 100, 1274-1284. [CrossRef]

41. Mo, Q.; Fu, A.; Deng, L.; Zhao, M.; Li, Y.; Zhang, H.; Feng, F. High-dose glycerol monolaurate up-regulated beneficial indigenous microbiota without inducing metabolic dysfunction and systemic inflammation: New insights into its antimicrobial potential. Nutrients 2019, 11, 1981. [CrossRef]

42. Zhao, M.; Cai, H.; Jiang, Z.; Li, Y.; Zhong, H.; Zhang, H.; Feng, F. Glycerol-monolaurate-mediated attenuation of metabolic syndrome is associated with the modulation of gut microbiota in high-fat-diet-fed mice. Mol. Nutr. Food Res. 2019, 63, 1801417. [CrossRef] [PubMed]

(C) 2020 by the authors. Licensee MDPI, Basel, Switzerland. This article is an open access article distributed under the terms and conditions of the Creative Commons Attribution (CC BY) license (http://creativecommons.org/licenses/by/4.0/). 